\section{Case Reports in Oncology}

\title{
Carcinoembryonic Antigen Elevation in a Patient with SARS-CoV-2 and a History of Colon Cancer
}

\author{
Taha Alrifai Anita Sharko William T. Leslie \\ Section of Medical Oncology, Division of Hematology/Oncology/Cell Therapy, Department \\ of Internal Medicine, Rush University Medical Center, Chicago, IL, USA
}

\section{Keywords}

SARS-CoV-2 - Colon cancer $\cdot$ Carcinoembryonic antigen

\begin{abstract}
The severe acute respiratory syndrome-coronavirus-2 (SARS-CoV-2) pandemic continues to affect millions of people and impact health-care delivery worldwide. New data and insights into the diagnosis and treatment of SARS-CoV-2 are emerging rapidly. Several prognostic biomarkers have been studied in patients with SARS-CoV-2. Among those biomarkers is the carcinoembryonic antigen (CEA). We report the case of a 46-year-old male with a history of colon cancer who was found to have an elevated CEA level during routine surveillance. Further workup confirmed a diagnosis of SARS-CoV-2 infection. The CEA level normalized with the resolution of the infection.
\end{abstract}

\section{Introduction}

The severe acute respiratory syndrome-coronavirus-2 (SARS-CoV-2) pandemic has impacted the delivery of medical care worldwide [1]. Our knowledge of SARS-CoV-2 is growing rapidly, including its impact on the management of oncology patients. Since its emergence, several biomarkers have been described as having prognostic and therapeutic implications in SARS-CoV-2 infections [2]. Recent studies have evaluated the carcinoembryonic antigen 
Fig. 1. Chest CT showing ground-glass opacities. CEA $3.5 \mathrm{ng} / \mathrm{mL}$. CT, computed tomography.

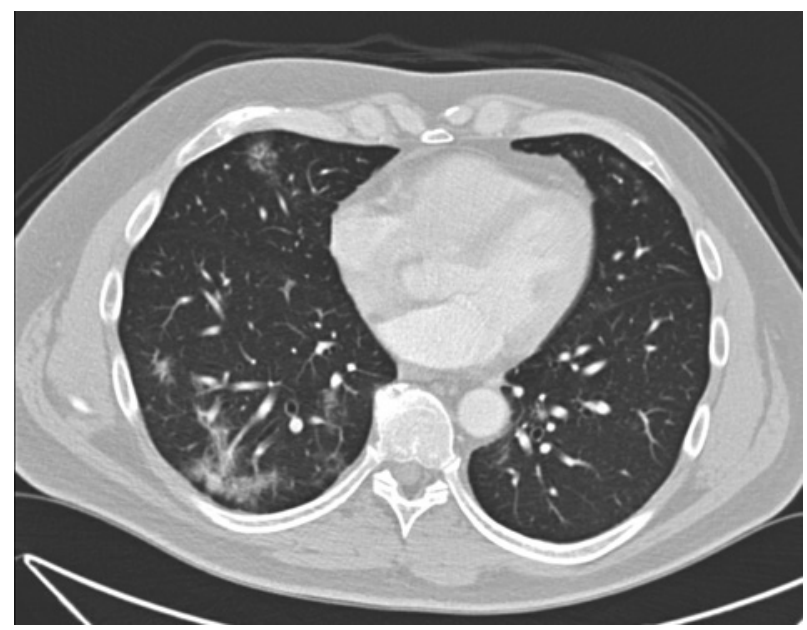

(CEA) as a prognostic biomarker in patients with SARS-CoV-2 [3, 4]. An elevated CEA level was associated with inferior outcomes. Herein, we describe a case of CEA elevation found during routine surveillance of a patient with stage IIIB colon cancer. This finding led to a diagnosis of SARS-CoV-2 infection. The CEA level normalized with the resolution of the infection.

\section{Case Report/Case Presentation}

We report the case of a nonsmoking 46-year-old male with a history of stage IIIB colon cancer, who was seen for routine follow-up and was found to have a slight elevation in the CEA level to $3.5 \mathrm{ng} / \mathrm{mL}$ (Reference range: nonsmokers $<3 \mathrm{ng} / \mathrm{mL}$, smokers $<5 \mathrm{ng} / \mathrm{mL}$ ). The CEA levels had been consistently $<3 \mathrm{ng} / \mathrm{mL}$ since the time of diagnosis. The CEA was $2.1 \mathrm{ng} /$ $\mathrm{mL} 3$ months ago. The patient had a moderately differentiated adenocarcinoma of the sigmoid colon resected 14 months ago, and 4 out of 25 lymph nodes were positive for metastatic carcinoma. He had received adjuvant folinic acid, fluorouracil (5-FU), and oxaliplatin (FOLFOX) chemotherapy. Given the newly elevated CEA level, a computed tomography scan was performed to evaluate for recurrence of the disease. This showed diffuse ground-glass opacities throughout the lungs (shown in Fig. 1), concerning for SARS-CoV-2 infection. It did not show evidence of colon cancer recurrence. A SARS-CoV-2 polymerase chain reaction test was performed, which was negative. 23 days later, SARS-CoV-2 serum IgG test was positive. A repeat computed tomography scan at that time showed significant improvement of the previously noted ground-glass opacities (shown in Fig. 2). The serum CEA level had decreased to $1.8 \mathrm{ng} / \mathrm{mL}$. This strongly suggested SARS-CoV-2 as the etiology of the elevated CEA level.

\section{Discussion/Conclusion}

A potential explanation for an elevated CEA in cases of SARS-CoV-2 is the fact that CEA is coded by the CEACAM5 gene, which is exclusively expressed by epithelial cells, including pneumocytes [5]. Increased CEA expression in pneumocytes has been detected in nonneoplastic pulmonary diseases $[3,6]$. The human angiotensin-converting enzyme 2 receptor is a known entry point for the SARS-CoV-2 virus and is abundantly expressed on the cell 
Fig. 2. Chest CT showing interval improvement in ground-glass opacities. CEA $1.8 \mathrm{ng} / \mathrm{mL}$. CT, computed tomography.

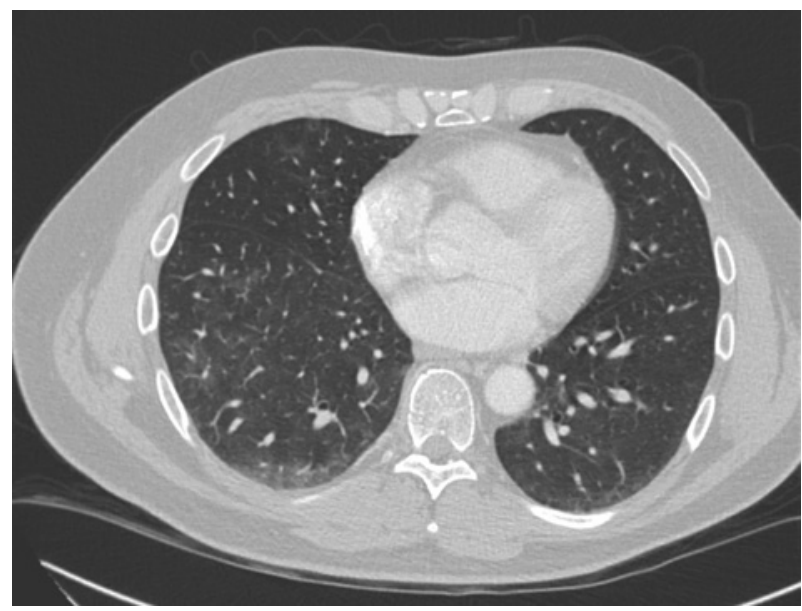

surface of pulmonary epithelial cells, allowing an entry point to pneumocytes with subsequent destruction of these cells. This is followed by regeneration of pneumocytes with a concordant increase in serum CEA levels [3].

Since the emergence of SARS-CoV-2, there have been attempts to identify prognostic serum biomarkers. Among those studied is the CEA. In a study by Yu et al. [4], 161 patients without a known history of cancer, who were admitted to acute care with a confirmed diagnosis of SARS-CoV-2 infection were found to have an elevated CEA level ( $>5 \mathrm{ng} / \mathrm{mL}$ ). The investigators found that higher levels of CEA were associated with a worse prognosis [4]. In another study that excluded patients with a known history of cancer, Chen et al. [3] evaluated the CEA level as a prognostic marker in patients with SARS-CoV-2. The study included 46 patients who died and 68 patients who were discharged from the hospital. The CEA level was significantly higher in the mortality group compared to the discharged group. Additionally, they found that the risk of death increased 1.317 times for every additional $1 \mathrm{ng} / \mathrm{mL}$ elevation in CEA.

To the best of our knowledge, our case is the first report of a CEA elevation in a patient with SARS-CoV-2 infection and a history of colon cancer. Oncologists should be aware of SARS-CoV-2 infection as an etiology of a CEA elevation in patients with a history of colon cancer. Also, additional studies investigating the role of CEA as a prognostic marker in SARSCoV-2 infections should be considered.

\section{Statement of Ethics}

Written informed consent was obtained from the patient for publication of this case report and any accompanying images.

\section{Conflict of Interest Statement}

The authors have no conflicts of interest to declare.

\section{Funding Sources}

The authors did not receive funding to support the conduction of this study.

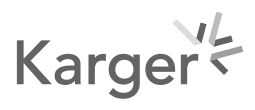




\section{Case Reports in Oncology}

\begin{tabular}{l|l}
\hline Case Rep Oncol 2021;14:802-805 \\
\hline DOI: 10.1159/000515261 & $\begin{array}{l}\text { @ 2021 The Author(s). Published by S. Karger AG, Basel } \\
\text { www.karger.com/cro }\end{array}$ \\
\hline
\end{tabular}

Alrifai et al.: CEA Levels in SARS-CoV-2 Infection

\section{Author Contributions}

Taha Alrifai: literature review and manuscript writing. Anita Sharko: conception of the work, drafting the manuscript, and revising it critically, final approval for the version to be published. William T. Leslie: conception of the work, drafting the manuscript and revising it critically, and final approval for the version to be published.

\section{References}

1 Jazieh AR, Akbulut H, Curigliano G, Rogado A, Alsharm AA, Razis ED, et al. Impact of the COVID-19 pandemic on cancer care: a Global Collaborative Study. JCO Glob Oncol1. 2020 Sep;6(6):1428-38.

2 Zhang L, Guo H. Biomarkers of COVID-19 and technologies to combat SARS-CoV-2. Adv Biomark Sci Technol2. 2020;2:1-23.

3 Chen Q, Kong H, Qi X, Ding W, Ji N, Wu C, et al. Carcinoembryonic antigen: a potential biomarker to evaluate the severity and prognosis of COVID-19. Front Med3. 2020;7:579543.

4 Yu J, Yang Z, Zhou X, Wu D, Chen J, Zhang L, et al. Prognostic value of carcinoembryonic antigen on outcome in patients with coronavirus disease 2019. J Infect4. 2020 Aug;81(2):e170-2.

5 Gao Y, Song P, Li H, Jia H, Zhang B. Elevated serum CEA levels are associated with the explosive progression of lung adenocarcinoma harboring EGFR mutations. BMC Cancer5. 2017 Jul;17:484.

6 Abbona GC, Papotti M, Gugliotta P, Pecchio F, Rapellino M. Immunohistochemical detection of carcinoembryonic antigen (CEA) in non-neoplastic lung disease. Int J Biol Markers6. 1993 Dec;8(4):240-3. 